Noname manuscript No.

(will be inserted by the editor)

\title{
Principle and experimental validation of a new apparatus allowing large deformation in pure bending: application to thin wire.
}

\author{
G. Antherieu - N. Connesson - D. Favier • \\ P. Mozer • Y. Payan \\ Received: / Accepted:
}

\begin{abstract}
Experimental pure bending conditions are difficult to obtain when large deformations and displacements are required. In this work, a new principle using two universal joints is proposed and developed to enable such pure bending conditions. This principle has been applied to design an apparatus suitable to test small size samples (such as wires of diameter $<1 \mathrm{~mm}$ ) at small curvature radii $(\simeq 5 \mathrm{~mm})$ and to specifically provide small size samples moment-curvature relationship. This article underlines and validates the abilities of this new apparatus by performing and analysing tests on samples made of well-known material.
\end{abstract}

Keywords Pure bending · Wire · Tube

\section{Introduction}

Various industries tend to employ miniaturized equipment which is particularly true in the medical field where devices such as stents, biopsy needles, coils, etc. are commonly used. These devices are mostly made up of thin components such as wire or tube, that are often subjected to bending load. The design of such devices thus requires the knowledge of these thin components momentcurvature relationship. This relationship is often obtained directly from experimental pure bending,

This work has been supported by the LABEX CAMI (Ref. ANR-11-LABX-0004).

N. Connesson

Laboratoire TIMC/IMAG, UMR CNRS 5525, Faculté de Médecine Domaine de la Merci, La Tronche, France Tel.:

Fax:

E-mail: nathanael.connesson@imag.fr 
or numerically deduced from conventional stress-strain relationship measured under uni-axial tension/compression. For most specimens, direct numerical estimation is impossible due to anisotropy, material heterogeneity, tension-compression asymmetry, etc. Moreover, the compressive material behaviour is usually not experimentally easy to obtain. An experimental means able to identify the moment-curvature relationship would thus be of great interest.

Bending experimental and theoretical approaches are well documented in the literature $[1,2$, 3], and are still getting significant attention [4]. Pure bending tests allow to identify the material moment-curvature relationship. In particular, different phenomena can be studied using bending measurements, such as tension/compression asymmetry [5] or localization in the sample [6]. However, if pure bending conditions are straightforward in numerical approach, achieving pure bending experiments is a thorny experimental problem, especially at large deformations. This work introduces and evaluates the principle of an original device to apply pure bending load. This device is intended to be used as a means to experimentally identify the moment-curvature relationship of slender specimens.

Pure bending is a loading configuration where the specimen is a beam bent in one of its planes of symmetry $P$ by two opposite, yet equal, couples $\vec{M}= \pm M \vec{z}$, where $\vec{z}$ is orthogonal to the plane of symmetry. Couples are applied to the ends B and C of the beam [1]. No other loads are applied to the specimen. In this configuration (Fig. 1,a):

- the concave part of the specimen is loaded in compression;

- the convex part of the specimen is loaded in tension;

- the neutral surface contains fibres that are not subjected to any tensile or compressive stress; the neutral axis is the intersection of the neutral surface with the plane of symmetry $P$;

- the overall resulting force $\vec{R}$ in the section $\mathrm{S}$ is zero and can be written:

$$
\vec{R}=\iint_{S} \underline{\underline{\sigma}} \vec{x} d S=\overrightarrow{0}
$$

where $\underline{\underline{\sigma}}$ is the Cauchy stress tensor, $\vec{x}$ the unit vector orthogonal to the cross-section $S$ and $d S$ is a surface area element; 
- the overall moment $\overrightarrow{M_{O}}$ at the point $O$ of the neutral axis is related to the stress in cross-section $S$ by:

$$
\overrightarrow{M_{O}}=M \vec{z}=\iint_{S}(y \vec{y}) \wedge(\underline{\underline{\sigma}} \vec{x}) d S
$$

where $\vec{y}$ is a unit vector contained in the cross-section $\mathrm{S}$ and orthogonal to $\vec{x}$. The plane defined by $(\vec{x}, \vec{y})$ thus represents the plane of symmetry $P$ afore mentioned.

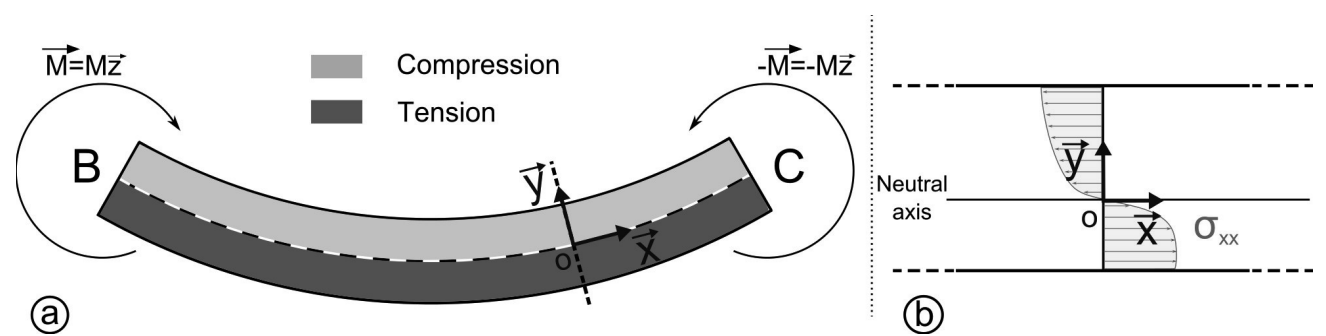

Fig. 1: a) Beam loaded in pure bending, b) Stress distribution in a section $\mathrm{S}$

Various apparatus were designed in the literature to generate pure bending loading $[7,8,9$, 10,4,11]. Most of them are using classical static 4-points bending configuration (Fig. 2,a). In this configuration only 4 forces $\overrightarrow{F_{A}}$ to $\overrightarrow{F_{D}}$ are applied to the specimen (Fig. 2,a): as long as displacements remain small, these forces are supposed to remain parallel and equal to each other.

As a consequence, the overall moment applied to the specimen expressed in eq. 2 can also be written:

$$
\overrightarrow{M_{O}}=-x F_{A} \vec{z}+(x-L) F_{B} \vec{z}=-\frac{F}{2} L \vec{z}
$$

This moment $\overrightarrow{M_{O}}=\frac{F}{2} l \vec{z}$ is uniform between B and C (Fig. 2, a); the moment does not depend upon the position of the cross section. Moreover, for small displacements, no tangential forces are observed at contact points $\mathrm{A}, \mathrm{B}, \mathrm{C}$ and $\mathrm{D}$, even though friction could occur, so that the resulting reaction force $\vec{R}$ is zero between $\mathrm{B}$ and $\mathrm{C}$.

For some materials (shape memory alloys -SMA- among others), large strain loading is required to fully characterize specimens. Moreover, these materials are sometimes only available as thin samples. A bending apparatus able to reach high strain $\left(\varepsilon_{x x} \sim 10 \%\right)$ on small size samples $(\sim 1 \mathrm{~mm}$ diameter) is thus required to test such materials, which implies reaching small curvature radii and allowing large displacement of the specimen extremities. With a conventional 4 points bending 
(a)

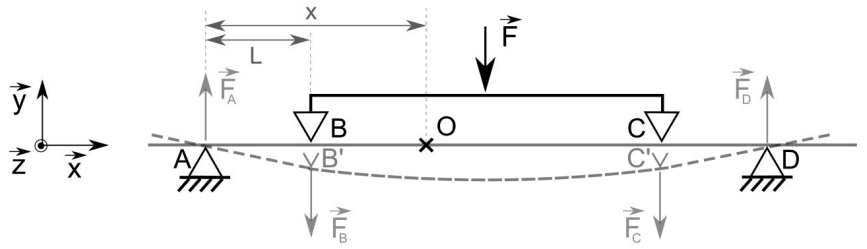

(b)
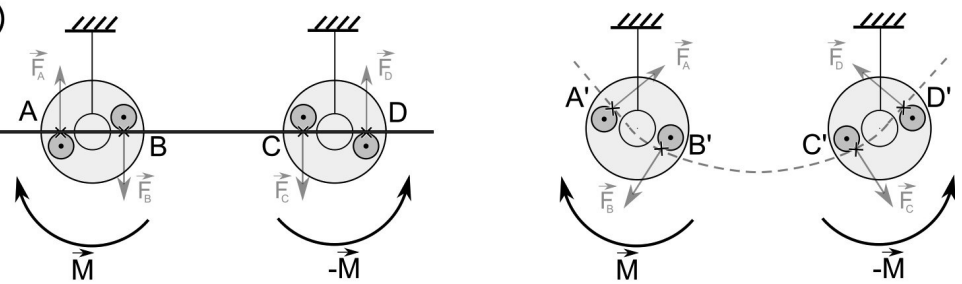

(C)
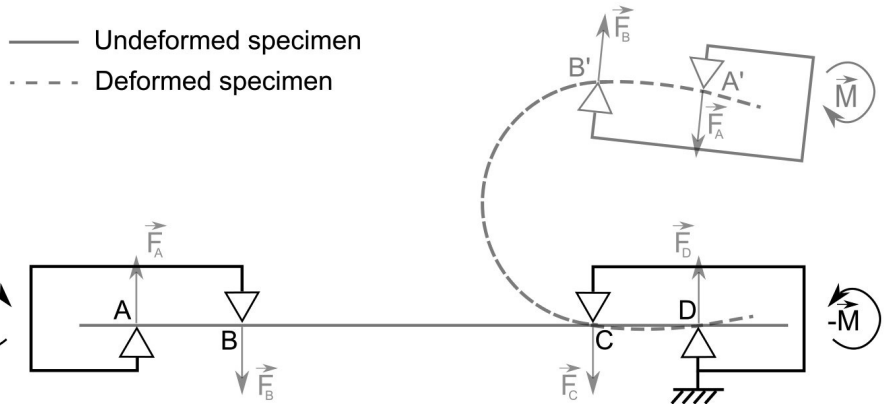

Fig. 2: Various bending devices: a) conventional 4-points bending, b) device developped by Reelunn et al.[4], c) apparatus presented in this paper

set-up such displacement would yet induce tangent friction forces at contact points $\mathrm{A}, \mathrm{B}, \mathrm{C}$ and D. In these conditions, the specimen would not be loaded in pure bending $(\vec{R} \neq \overrightarrow{0}$, eq 1$)$. This limitation applies to numerous other devices which are dedicated to pure bending $[11,9,10]$.

According to the authors, the pure bending device designed by Kyriakides et al. is the most appropriate to meet the required set-up characteristics. Reedlunn et al. miniaturized this system, leading to a bending apparatus able to provide results on smaller samples (tubes of $3.176 \mathrm{~mm}$ outer diameter) [4]. This apparatus is a 4-points bending device using four rollers mounted on two loading wheels (Fig. 2,b). This system overcomes the limitation of conventional 4-point bending machines: the use of rollers enables the specimen to slide along its own axis. Tangential force elements are thus maintained close to zero; the specimen slides until it reaches its equilibrium position, so that the resulting reaction force $\vec{R}$ is zero (eq. 1). Yet, this system presents a limited curvature range 
radii due to the constant distance between the two loading wheels. Moreover, the sliding of the specimen leads to a variation of the loaded length during the testing.

To circumvent these limitations, an innovative pure bending principle has been proposed to keep a constant loaded length during testing, and to reach a wide range of curvatures. This principle has been used to design an apparatus able to reach $5 \mathrm{~mm}$ radius of curvature from a initial linear geometry, with wire diameters of less than $1 \mathrm{~mm}$.

The principle is also based on a 4-points mechanism (Fig. 2,c): one grip is static (points $\mathrm{C}$ and D) while the other grip (points A and B) can freely move in every directions. This mobile grip only applies the couple $\overrightarrow{M_{O}}$ to the sample through the contact points $\mathrm{A}$ and $\mathrm{B}$. As the mobile grip applies no resulting load to the specimen, the resulting reaction force $\vec{R}$ in the specimen cross section is zero (eq. 1). This insures both pure bending and the possibility to reach small curvature radii. To our knowledge no device using this technical solution or presenting the same ability has ever been presented in the literature.

This paper presents the apparatus (section 2), the validation process (section 3), and preliminary experimental bending results (section 4).

\section{Experimental set-up}

\subsection{Pure bending apparatus}

An original pure bending apparatus has been developed. The technical solutions chosen to obtain the required mobility of the moving grip are presented in Fig. 3. As a rough description:

- the bending couple $\vec{M}$ is applied to extremity $\mathrm{H}$ of shaft 1 ;

- the required grip mobilities are insured by two universal joints (Fig. 3,a) located at both extremities of shaft 2 . These mechanical linkages transmit the bending load, and minimize both shear and torsion in the specimen;

- the ball bearing at the specimen extremity also minimizes torsion and shear loads in the specimen;

- using a counterweight, the centre of gravity of the shaft 2 is designed to be localized at the centre of the universal joint. This prevents the specimen from being loaded with shaft 2 own weight. 
a)

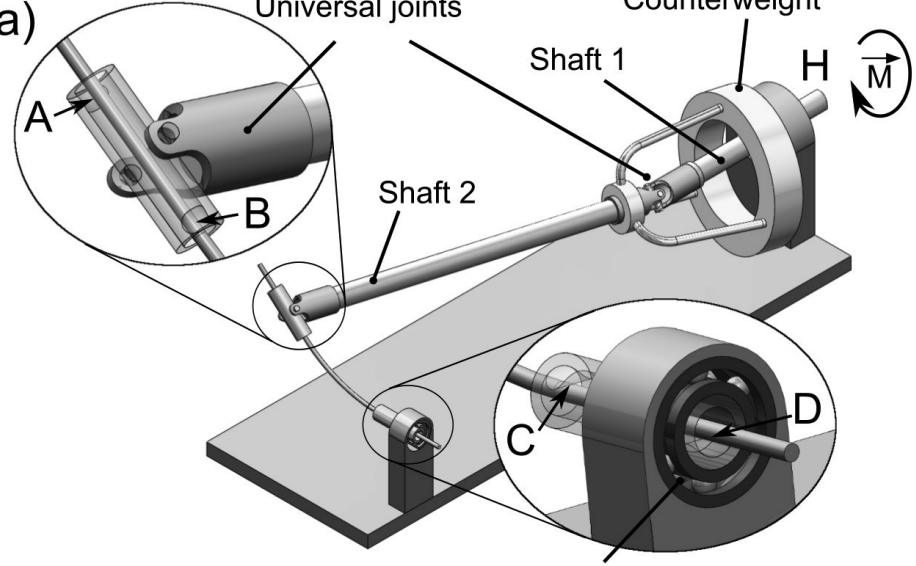

b)
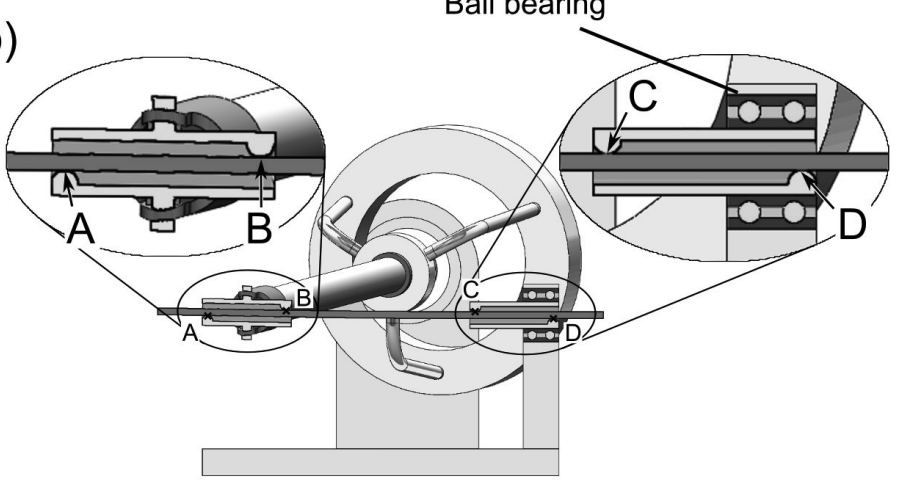

c)

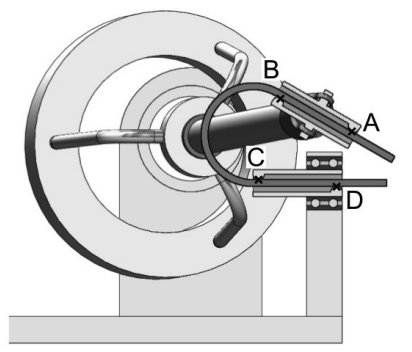

Fig. 3: Bending apparatus: a) $\frac{3}{4}$ view, b) and c) front views in different positions during a bending test.

Two extremal specimen positions along with sectional views of the grips and specimen extremities are presented Fig. $3 \mathrm{~b}$ and c. The miniaturized apparatus used in this work can fit in a $60 \mathrm{~mm} \times 90 \mathrm{~mm} \times 100 \mathrm{~mm}$ volume. The principle of this pure bending apparatus has been patented [12]. 


\subsection{Measurement methods}

During the bending test, both the bending moment $\overrightarrow{M_{O}}$ (section 2.2.1) and the curvature radius $R$ (section 2.2.2) are measured. In the following part, the measurement methods are presented along with the related errors.

\subsubsection{Bending load measurement}

In this study the bending moment $\overrightarrow{M_{O}}$ is produced by an actuator wire pulling a pulley (not drawn on the figure). The wire is translated using a Gabo Eplexor 500N tensile machine. The wire load is measured using a $25 \mathrm{~N}$ load cell. A preliminary calibration study provided an error on the moment $\overrightarrow{M_{O}}$ estimated to be $1.10^{-4} \mathrm{Nm}$.

\subsubsection{Curvature measurement}

The specimen deformed shape between point B and C is a perfect arc of circle during the loading if the following hypotheses are fulfilled:

- the specimen is loaded in pure bending (Hyp. H1);

- the initial shape of the neutral fibre is a straight line or a circle, with initial curvature radius $R_{0}$ (Hyp. H2);

- the material is homogeneous between B and C (Hyp. H3);

- the cross section is identical in each section between B and C (Hyp. H4).

It should be underlined here that the resulting specimen shape should remain a circle even if the material mechanical behaviour would be non-linear or asymmetrical in tension and compression.

In this work, the shape of the specimen between points $\mathrm{B}$ and $\mathrm{C}$ has been emphasized by studying the local curvature radii variations. The following section details the applied methodology.

Pictures were taken during the test and synchronized with the load measurement $\overrightarrow{M_{O}}$. The specimens local curvatures were then estimated along the specimens using these pictures (Fig. 4). Different steps are required:

- The median line of the sample is identified, providing several median points $P=\left(p_{1}, \ldots, p_{n}\right)$

(Fig. 4).The coordinates of points $p_{i}$ are noted $\left(x_{i}, y_{i}\right)$; 
- the identified radius $R_{i d}^{j}$ is computed locally on a mobile window containing $2 K+1$ points, and centred on a point $p_{j}$;

- using these points, a circle is fitted in the least mean-square sense. The distance between the circle and a point $p_{i}$ of the mobile window is written:

$$
d_{i}^{j}\left(X_{o}, Y_{o}, R_{i d}^{j}\right)=||\left|\overrightarrow{O p_{i}}\right|\left|-R_{i d}^{j}\right|=\left|\sqrt{\left(Y_{o}-y_{i}\right)^{2}+\left(X_{o}-x_{i}\right)^{2}}-R_{i d}^{j}\right|
$$

where the unknowns are the circle centre coordinates $X_{o}, Y_{o}$ and its radius $R_{i d}^{j}$. These unknowns

are estimated in the least mean square sense by minimizing the function $\Phi=\sum_{i=j-K}^{j+K} d_{i}^{j^{2}}\left(X_{o}, Y_{o}, R_{i d}\right)$.

Local curvature radii $R_{i d}^{j}$ are estimated along the whole specimen by moving the mobile window point after point. The window size $2 K+1$ is adjusted so that the window length is at least two times the specimen thickness.

\section{Numerical validation of radius measurement}

Radius measurement requires the evaluation of a second derivative and is thus very sensitive to noise. This is inherent to experimental conditions such as lighting, image resolution/pixelation, etc. that induce noise on median points $p_{i}$ identification. The noise sensitivity to image resolution/pixelation has been numerically estimated on two cases:

- dealing with uniform curvature radius on a numerical circle;

- dealing with non-uniform curvature radius on a numerical spiral.

Perfect arc of circle and spiral numerical pictures were created (Fig. 4, a and c). Pixel size was chosen to be $1 p x=0.0125 \mathrm{~mm}$ so as to mimic pictures obtained experimentally. The analytical spiral local curvature radius function can be written:

$$
R_{\text {spiral }}=\alpha \theta+\beta
$$

where $\alpha$ is the radius increasing rate and $\beta$ is the initial radius. The chosen values for $\alpha, \beta$, the circle radius $R_{\text {circle }}$ and the line thickness are reported in Table 1.

The identified local curvature radii $R_{i d}$ along the curvilinear abscissa of the specimen $s(m m)$ are presented Fig. 4 b,d. Concerning the numerical circle, the method was able to identify $R_{i d}$ 


\begin{tabular}{|c|c|c|c|}
\hline$R_{\text {circle }}$ & $\alpha$ & $\beta$ & Line thickness \\
\hline $5 \mathrm{~mm}$ & $\frac{6.25}{\pi} \mathrm{mm}$ & $3.125 \mathrm{~mm}$ & $0.5 \mathrm{~mm}$ \\
\hline
\end{tabular}

Table 1: Numerical circle and spiral parameters

with a mean relative error of $2.410^{-3} \mathrm{~mm}$ and corresponding standard deviation of $1.010^{-2} \mathrm{~mm}$ in comparison with the circle radius $R_{\text {circle }}$ (Fig. 4,a). When curvature radius is not uniform (numerical spiral), error $e_{r}$ associated with the local curvature radius estimation remains lower than $1 \%$ (Fig. 4,b). Corresponding mean relative error was measured as $9.910^{-3} \mathrm{~mm}$ with a standard deviation of $1.410^{-2} \mathrm{~mm}$.

A global radius estimate can also be identified on the specimen by considering all the identified median points $p_{i}$ in the window. With this setting, the circle global curvature radius $R_{\text {global }}$ has been identified as $R_{\text {global }}=4.996 \mathrm{~mm}$ (error of $0.08 \%$ ) on the numerical circle picture.

For curvature radii higher than $\beta=3.125 \mathrm{~mm}$, local curvature radius identification errors are thus considered negligible and independent of the curvature; these results confirm the ability of the method to estimate local curvature radii along specimens.

\section{Experimental pure bending validation}

Since the performances of our measurement method have been evaluated, the apparatus ability to apply pure bending load to specimens has now to be demonstrated: experiments on well-known materials were thus performed for validation. These tests aim at demonstrating the validity of hypothesis H1 (section 2.2.2).

Specimens were selected so as to fulfil hypotheses H2, H3 and H4. In these conditions, regardless the mechanical behaviour of the tested material, if a pure bending test is achieved (H1), then:

- the specimen curvature should be uniform between points B and C (circular shape) (i);

- experimental and theoretical bending moments should be equal (eq. 2) (ii);

In this section, assessments (i) and (ii) were checked on two materials presenting very different mechanical behaviours: work-hardened steel and annealed copper specimens. 

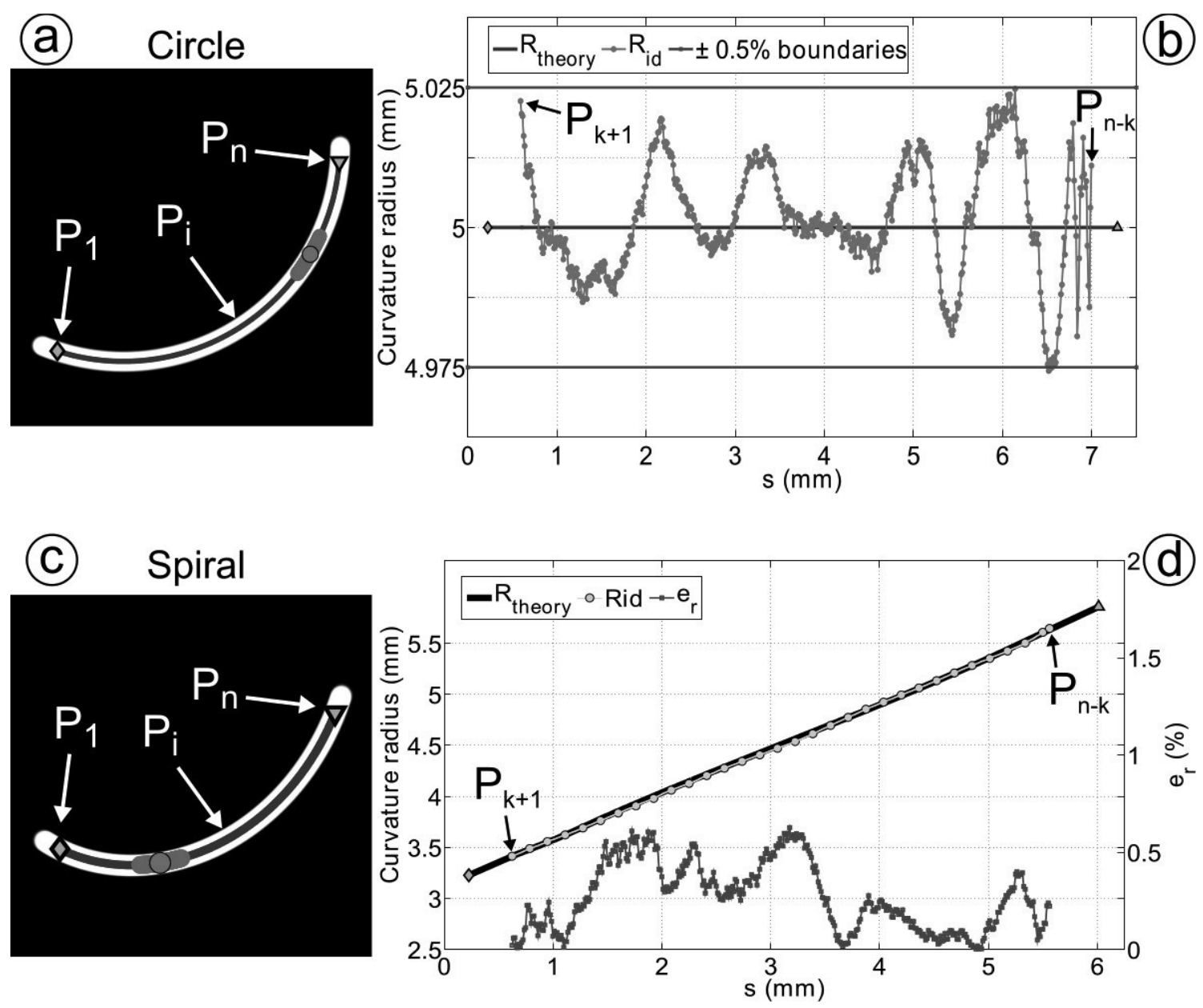

Fig. 4: Numerical pictures with identified median points (thin blue line) and a typical mobile window (thick blue line) centred on a median point (red spot), along with estimated $R_{i d}$ and corresponding error $e_{r}:$ a) and b) circle, c) and d) spiral.

\subsection{Steel specimens}

A first bending test has been performed using work-hardened steel (not annealed) wires (diameter $0.5 \mathrm{~mm}$ ) at room temperature (Fig. 6).

The local fitting method described previously has been applied on various pictures to examine the local curvature along the sample during the bending test (Fig. 5). Results underline the local curvature uniformity along the sample at different stages of loading. This uniformity confirms the pure bending hypothesis H1. 
For further validation, the material elastic modulus deduced from the pure bending test has been compared to the elastic modulus identified during the uni-axial tension test.

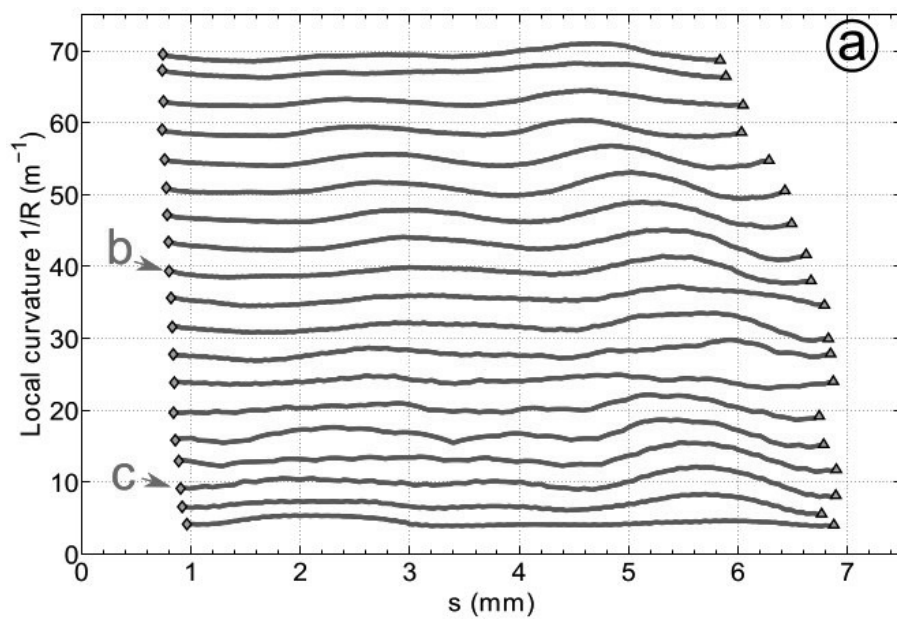

(a)
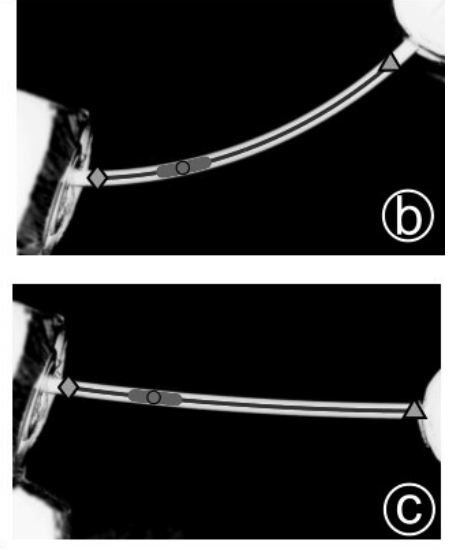

Fig. 5: Steel specimen-local fitting method used to check the local curvature uniformity: a) Local curvature along the sample for various pictures, b) A typical result, with the identified median points $p_{i}$ (thin blue line) located between the first point $p_{1}$ (green diamond) and the final point $p_{n}$ (green triangle), along with a typical computing window (thick blue line) centred on a median point $p_{j}$ (red spot), c) Another typical result.

Under Bernoulli hypotheses, and taking the origin of the coordinates at the neutral axis of the cross section (Fig. 1,b), the strain gradient in the section can be written:

$$
\varepsilon_{x x}=y\left(\frac{1}{R}-\frac{1}{R_{0}}\right)
$$

where $R_{0}$ and $R$ are the initial and current curvature radii respectively. It should be underlined here that equation 6 remains valid regardless the material mechanical behaviour.

In the specific case of a linear-elastic behaviour, the bending moment $\overrightarrow{M_{O}}$ can be easily deduced from eq. 2 and $6[1]$ :

$$
\overrightarrow{M_{O}}=E I\left(\frac{1}{R}-\frac{1}{R_{0}}\right) \vec{z}
$$

where $E$ is the Young's modulus and $I$ the second moment of area. Steel specimen tensile behaviour was determined experimentally using a Gabo Eplexor 500N machine with a $500 \mathrm{~N}$ load cell at nearly isothermal strain rate in room temperature (Fig. 6,a): 
- Young modulus found during the tensil test is $E_{\text {steel }}^{\text {Tension }}=200 \mathrm{GPa}$;

- offset yield point was taken at $0.2 \%$ plastic strain. The corresponding offset yield stress and strain are $R_{p 0.2}=1.66 G P a$ and $\varepsilon_{p 0.2}=1.03 \%$

In the linear-elastic domain, the experimental bending load increases linearly with curvature $\left(\frac{1}{R}\right)$ as predicted by the theory (Fig. 6,b):

- results show good linearity, both during loading for $M_{O}<M_{p 0.2}=0.021 \mathrm{Nm}$ and unloading;

- the identified Young modulus with the experimental moment is $E_{\text {steel }}^{\text {Bending }}=205 G P a$; this value is consistent with the literature [13] and with the experimental tensile behaviour, even if the use of bending tests are usually not recommended to measure elastic moduli,

- conjectured offset yield curvature $C_{p 0.2}=33 \mathrm{~m}^{-1}$ was determined from tensile behaviour, for curvatures $\frac{1}{R}>C_{p 0.2}$ the elasto-plastic domain is reached, and the bending moment $\overrightarrow{M_{O}}$ is no longer linear with curvature: the sample is plastically strained.
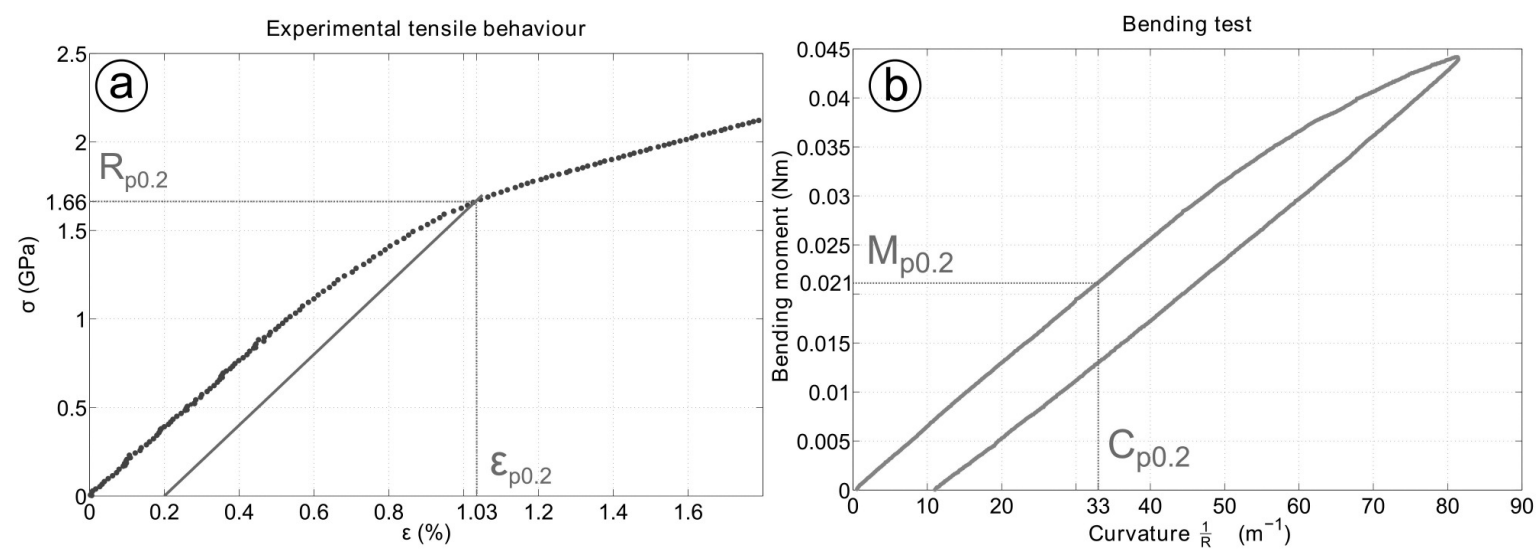

Fig. 6: a) Tensile testing on work-hardened steel with offset yield point at $0.2 \%$ plastic strain, b) Bending testing on work-hardened steel.

As expected, the measured moment $\overrightarrow{M_{O}}$ variation is in accordance with the theory.

\subsection{Copper specimens}

Experiments have also been performed on soft annealed copper wires (diameter $0.6 \mathrm{~mm}$ ) at room temperature (Fig. 8). 
Again, the local fitting method have been used to check the local curvature uniformity along the sample at different stages of loading (Fig. 7). At low curvatures, experimental lighting defects unfortunatly led to a shift in the identification of median points $p_{i}$, which biased the local curvatures estimation ( $c f$. section 2.2.2). Nevertheless, local curvature uniformity along the sample at different stages of loading confirms the pure bending hypothesis H1.

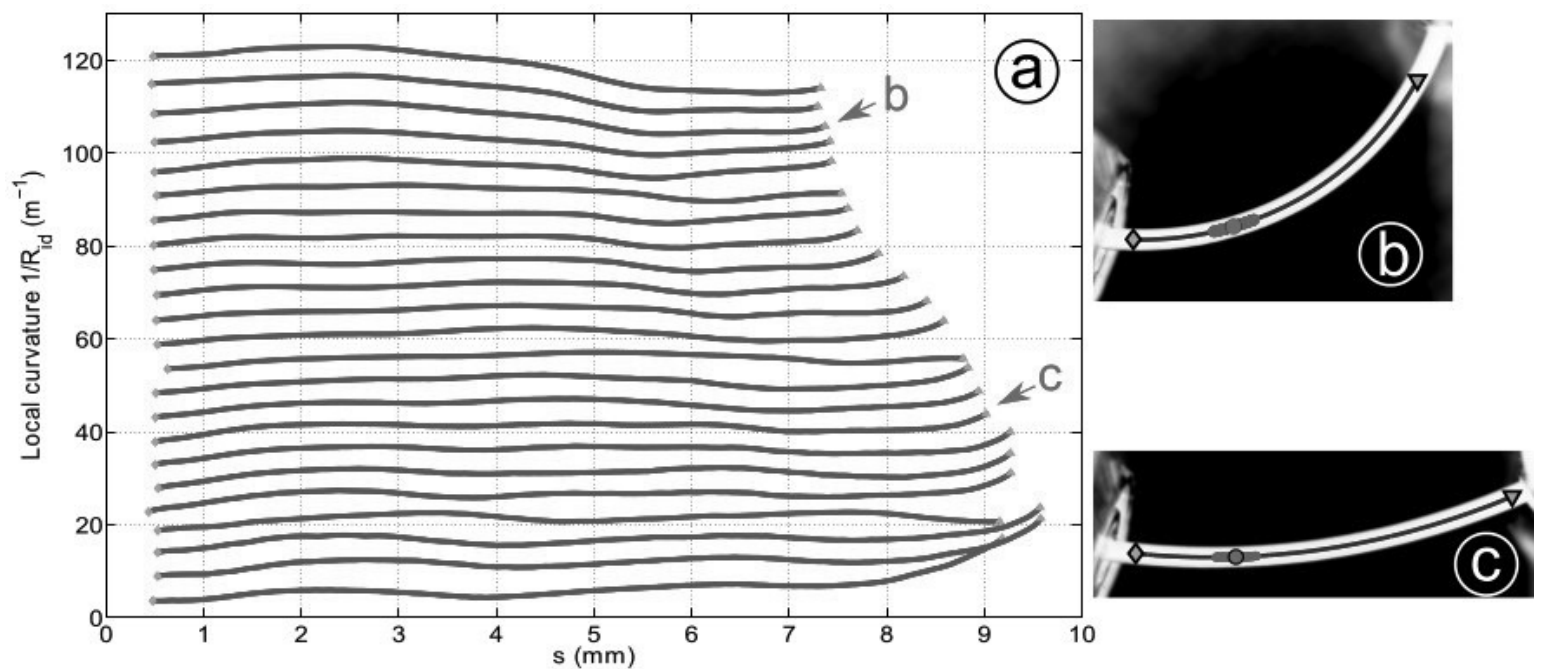

Fig. 7: Copper specimen-local fitting method used to check the local curvature uniformity:: a) Local curvature along the sample for various pictures, b) A typical result, with the identified median points $p_{i}$ (thin blue line) located between the first point $p_{1}$ (green diamond) and the final point $p_{n}$ (green triangle), along with a typical computing window (thick blue line) centred on a median point $p_{j}$ (red spot), c) Another typical result.

Although the specimens were loaded beyond the linear elastic field, the general bending moment expression given in eq 2 remains valid. The tensile behaviour of the specimen $\sigma_{x x}^{\text {tension }}(\varepsilon)$ was determined experimentally using a Gabo Eplexor 500N machine with a 500N load cell, during a quasi-static isothermal test at room temperature (Fig. 8, a). Specimen mechanical behavior has been assumed to be symmetrical in tension-compression [14]. The conjectured bending moment versus curvature has simply been calculated using eq 2 , knowing the $\sigma_{x x}(\varepsilon)$ function in tension and compression. Comparison between the conjectured and experimental moment $\overrightarrow{M_{O}}$ is presented in Fig. 8. Corresponding mean relative error was found to be $-1.810^{-5} N m$ with a standard deviation 
(a)

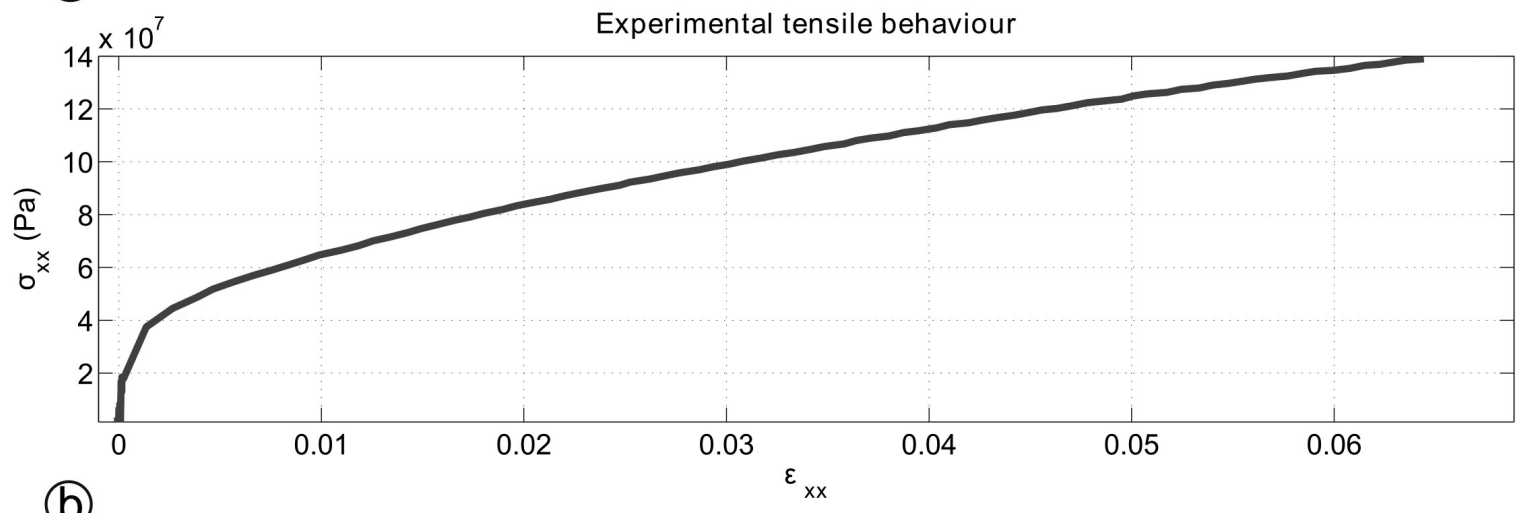

(b)

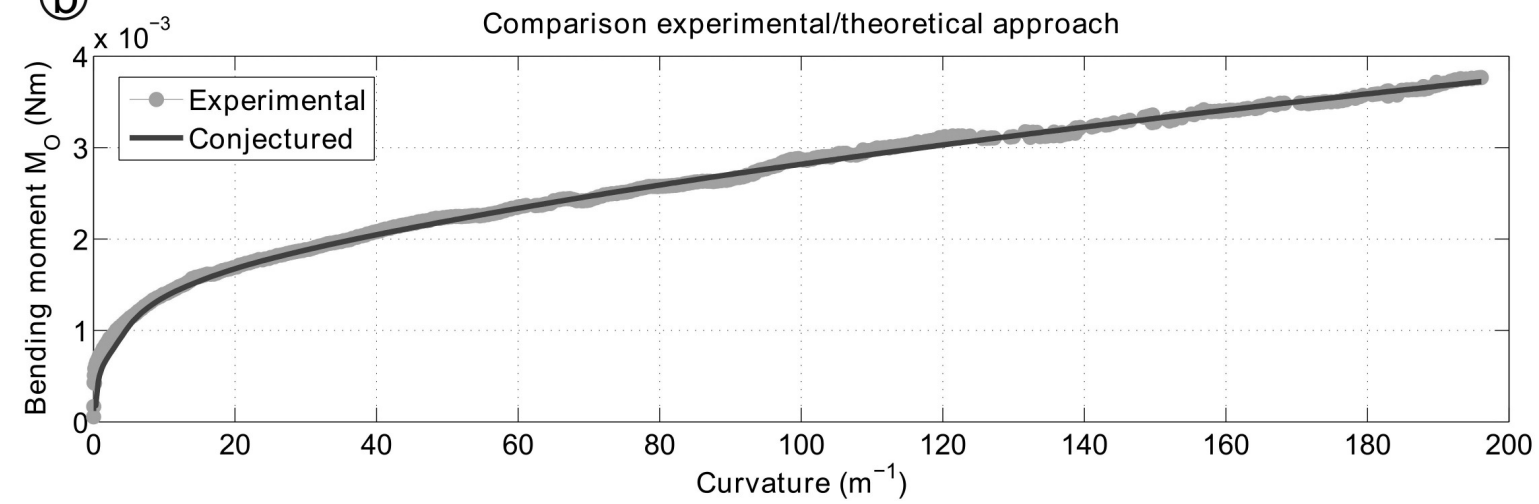

Fig. 8: Bending test on copper: comparison between experimental and theoretical results

of $5.710^{-5} \mathrm{Nm}$. This error is one order of magnitude less than the error on bending moment measurement $\left(10^{-4} \mathrm{Nm}\right)$.

These experimental results thus confirm the ability of our device to apply pure bending moments to specimens.

\subsection{Discussion}

Experimental results afore-presented validate the apparatus potency to be used as a means to access moment-curvature relationship of slender specimens even if large displacements and deformation are required. To improve the current device, the load cell sensitivity could be enhanced, enabling a more accurate bending moment estimation. Moreover, the quality of the experimental pictures could be improved by addressing the following points: 
- enhancement of the camera resolution;

- improvement of the lighting conditions so as to improve the identification of the median points $p_{i}$ (issues presented in section 4.2).

These modifications would reduce the related error of curvature identification.

\section{Conclusion}

An innovative principle to perform pure bending at high deformation and allowing large displacements for specimen extremities has been proposed. This principle has been applied and an apparatus has been developed and tested. This apparatus is a tool to experimentally identify material moment-curvature relationship. The errors associated with the apparatus and with the measurement methods are estimated to be: $10^{-4} \mathrm{Nm}$ on the bending moment measurement, and $0.08 \%$ on the global radius of curvature. The bending apparatus has been tested on small samples (wires with a diameter of $0.5 \mathrm{~mm}$ ) made of conventional work-hardened steel and copper. In both cases the curvature uniformity along the sample proved the device ability to apply pure bending load to specimens. The measured bending moments versus curvature were also analysed and proved to be consistent with literature and theory. The apparatus was thus able to provide reliable experimental results at small curvature radii $(5 \mathrm{~mm})$. This pure bending apparatus will be used for further investigations, such as determining specific material behaviour in bending.

\section{References}

1. S Timoshenko and JN Goodier. Theory of Elasticity. McGraw-Hill Book Company, 1951.

2. J Dryden. Bending of inhomogeneous curved bars. International Journal of Solids and Structures, 44(1112):4158-4166, June 2007.

3. SG Lekhnitskii. On the bending of a plane inhomogeneous curved beam. Journal of Applied Mathematics and Mechanics, 43(1):182-183, 1979.

4. B Reedlunn, CB Churchill, EE Nelson, JA Shaw, and SH Daly. Tension, compression, and bending of superelastic shape memory alloy tubes. Journal of the Mechanics and Physics of Solids, 63:506-537, February 2014.

5. L Orgéas and D Favier. Stress-induced martensitic transformation of a NiTi alloy in isothermal shear, tension and compression. Acta Materialia, 46(15):5579-5591, 1998.

6. NJ Bechle and S Kyriakides. Localization in NiTi tubes under bending. International Journal of Solids and Structures, 51(5):967-980, March 2014. 
7. G Arnold, S Calloch, D Dureisseix, and R Billardon. A pure bending machine to identify the mechanical behaviour of thin sheets. In 6th International ESAFORM Conference on Material Forming, pages 1-4, 2003.

8. L Orgeas and D Favier. Application of the Beam Theory to Model the Pseudoelastic and Ferroelastic Bending of SMA Beams. Journal de physique IV, 5:2-7, 1995.

9. S Kyriakides and GT Ju. Bifurcation and localization instabilities in cylindrical shells under bending - I Experiments. International Journal of Solids and Structures, 29(9):1117-1142, 1992.

10. T Ghanbari Ghazijahani and H Showkati. Experiments on cylindrical shells under pure bending and external pressure. Journal of Constructional Steel Research, 88:109-122, September 2013.

11. JPM Hoefnagels, CA Buizer, and MGD Geers. A miniaturized contactless pure-bending device for in-situ SEM failure analysis. Experimental and Applied Mechanics, 6:587-596, 2011.

12. Nathanael Connesson, Gabriel Antherieu, Denis Favier, and Yohan Payan. Patent n FR1456479: Dispositif de test mécanique en flexion pure et procede de mise en oeuvre, 2014.

13. D McClaflin. Torsional deformation and fatigue of hardened steel including mean stress and stress gradient effects. International Journal of Fatigue, 26(7):773-784, July 2004.

14. Rolf Sandström, Josefin Hallgren, and Gunnar Burman. Stress strain flow curves for Cu-OFP, R-09-14, 2009. URL: www.skb.se. 